\title{
URI Online Judge Academic: A Tool for Professors
}

\author{
Jean Luca Bez ${ }^{1}$ Carlos E. Ferreira ${ }^{2}$ Neilor A. Tonin ${ }^{1}$ \\ ${ }^{1}$ Computer Science Department, Universidade Regional Integrada, Erechim, RS, Brazil \\ ${ }^{2}$ Computer Science Department, Institute of Mathematics and Statistics, University of \\ São Paulo, Brazil
}

\begin{abstract}
There is an increasing demand for tools that help the learning process in all areas of knowledge. The students have more and more difficulties with the traditional classes based on the exposition of the contents in the blackboard or slides. We need new methods and tools to better explore the new technologies available. The URI Online Judge is such a new tool, specially focused on teaching Algorithms and Programming. The work presented here describes the URI Online Judge Academic, a specific module, which allows the professor a greater control of disciplines (groups) and exercises and it allows them to track the students' progress in an organized and intuitive online environment.
\end{abstract}

Keywords: academic tool, online judge, algorithms, programming practice.

\section{Introduction}

This paper presents the URI Online Judge Academic, an online tool to help courses on Algorithms and Programming. The main goal of the URI Online Judge Academic is to provide an online platform that allows professors to track of the students' progress on the course.

The paper is structured as follows: In Section 1, we give an overview of the URI Online Judge website. In Section 2, we present the main reasons that motivated the Academic module, the main features available in this new tool, and how they can help both professors and students inside and outside of the classroom. Finally, in Section 3 we present some data about its use so far.

\section{The URI Online Judge}

The URI Online Judge is a project that is being developed at URI - Universidade Regional Integrada, Brazil. It is a website with problems in the style of the ICPC (International Collegiate Programming Contest) of the ACM, which provides the user an online judge to test his/her solutions to those problems. The first public presentation of the website was in July 2012 at WorldComp'12 [1]. Since then, the portal has undergone several improvements and incorporated new features to better suit the needs of all its users.

The aim of the work developed is to provide an online tool that can be used both by professors, in order to complement their lectures, and by students, to practice the contents seen in the classes. There are several benefits by incorporating the tool in the course. First, it introduces a new alternative to the traditional teaching method [2]. Furthermore, since many different problems are available, the student is encouraged to learn new subjects, not restricted to the content taught in the classroom. 
Coaches of teams that are preparing to participate at the ICPC can also use the tool. The coach may suggest interesting problems or subjects, and students are able to test their solutions by themselves.

A first feature of the URI Online Judge is the division of problems into eight major categories as illustrated by Fig. 1, thus allowing the student to better focus their studies. Another feature of the portal is the Toolkit: the user may send a test case, and the system automatically generates the corresponding correct output. It allows the student to understand better the problem and it provides a tool to test his/her solutions.

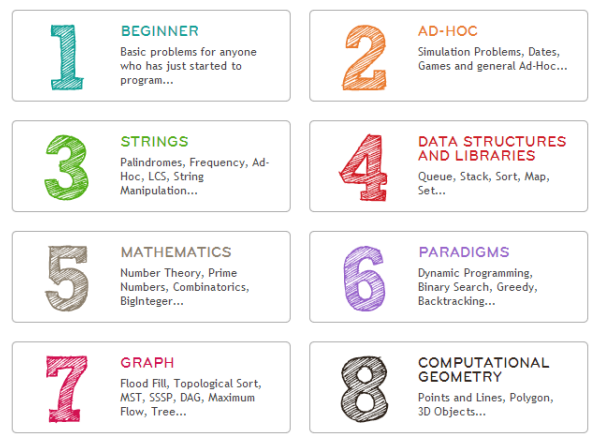

Fig. 1: URI Online Judge Categories.

One of the new features available in the latest release is the visualization of source codes previously submitted to the tool directly from the browser window.

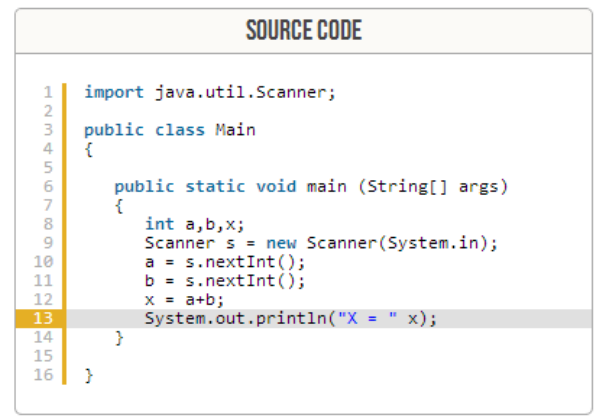

Fig. 2: Compilation Error.
For those submissions judged as containing "Compilation Errors", all lines with errors are highlighted, as shown in Fig. 2. Therefore, the student can visually observe what actually happened with the code when it was compiled and run by the Judge.

\section{The URI Online Judge Academic}

After six months using the Online Judge, Judge, many users, such as professors and ICPC coaches realized the need for a new environment. This should integrate all the necessary data to allow a better visualization and monitoring the progress of each student. That need resulted in the development of the URI Online Judge Academic.

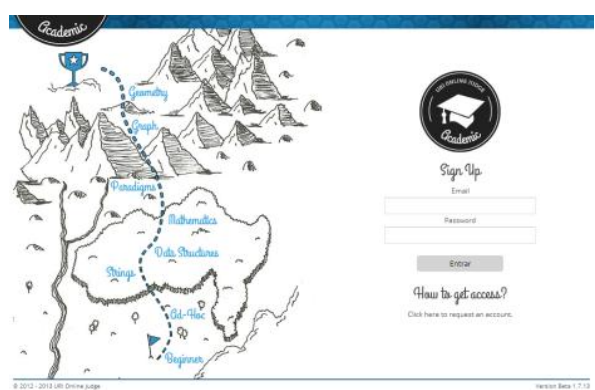

Fig. 3: URI Online Judge Academic

The access to the Academic module is granted only for professors, after his/her identity is confirmed. This is done to protect the users' privacy. Once the access is granted, he/she can enjoy all the features and facilities available. With the account, it is possible to structure groups or disciplines (via invitations). The professor creates a new group and invites his/her students to participate. By using the Academic, the professor may help students who have more difficulties to understand the subjects, while, at the same time, other students are free to explore. This structure enables the 
students to improve their skills in problem solving and in programming.

In each discipline or group, it is possible to create homework assignments, as many as needed, by selecting any problem of the URI Online Judge Problem Repository. Each of these lists may contain up to 20 exercises (problems). At the time of its creation, a deadline is set from when the system will no longer score if the student solved or not the proposed exercises. This way the professor can evaluate the participation and performance of each student.

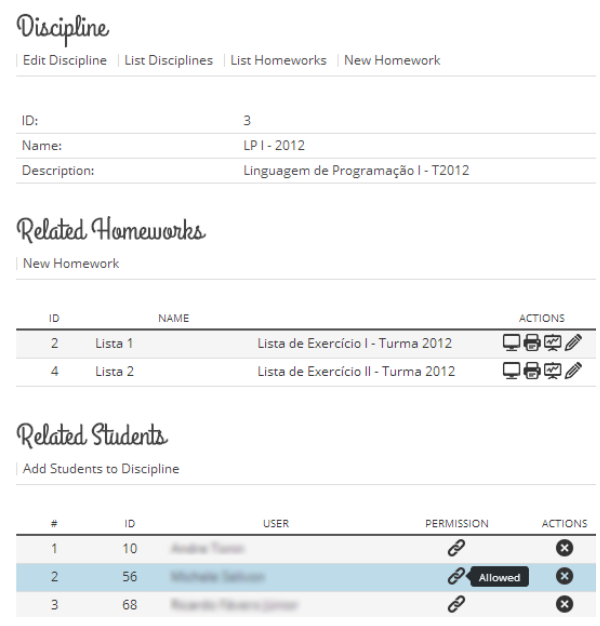

Fig. 4: Discipline / Group.

The Repository contains problems of levels that vary from 1 (easy) to 10 (hard). To add them to the homework (Fig. 5), one should just select those that best fit to the content studied in the class.

\section{Related Exercises}

New Exercise

\begin{tabular}{cclc} 
ID & & \multicolumn{1}{c}{ PROBLEM } & LEVEL \\
\hline 1 & 1192 & Paula's Mathematic Game & 2 \\
\hline 2 & 1193 & Base Conversion & 3 \\
\hline 3 & 1199 & Simple Base Conversion & 2 \\
\hline 4 & 1026 & To Carry or not to Carry & 2
\end{tabular}

Fig. 5: Problems selected for a homework.
The Progress Table is a table that allows the professor to track the progress of each student, knowing when it was the first time he/she tried to solve a problem from the list and if he/she succeeded in solving it. The data presented in that table follow the same pattern as in Fig. 6 .

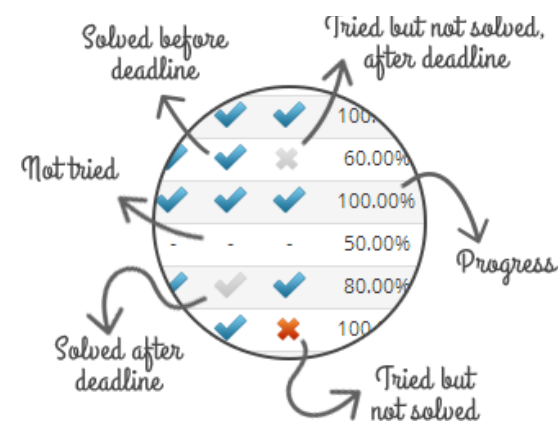

Fig. 6: Progress Table Icons.

Because the Academic module is fully integrated with the portal, the students will have immediate access to the disciplines and homework assignments as soon as they accept the invitation. Therefore, all disciplines and homework are private, that is, no other professor or student can access it.

The tool makes it easier to produce new assignments, monitor each student individual performance, and correct the exercises. Since the codes are available for the professor, he/she can evaluate them, to assure that the student solved the problem by himself, without using other codes available in the internet. All those features are available in an organized and intuitive interface.

\section{Statistics}

The main reason for the recent growth in the number of users of the URI Online Judge is that several professors from various universities in Brazil are using the Academic module. They incorporated the tool in their lectures as a way to allow 
the student to practice and apply the concepts learned in practical problems.

The Academic module already has about 60 professors enrolled, 70 disciplines (groups created) and more than 95 valid homework assigments. Of all the 4200 portal users, about 470 students already have access to those assignments created by their professors.

\section{Conclusions}

The Academic module complements the URI Online Judge website providing the professor (or coach of an ICPC team) an easy to use tool to construct assignments, monitor the students' performances and correct the exercises. The professor may easily detect students with more difficulties and help them, and, at the same time, advanced students are free to explore the repository, solving harder problems on the subject or even learning further topics.

\section{References}

[1] TONIN. Neilor A.; BEZ. Jean Luca, URI Online Judge: a new classroom tool for interactive learning, in FECS proceedings at WORLDCOMP'12, pp. 242-246.

[2] TONIN, N.A., ZANIN, F.A.; BEZ, J.L., Enhancing traditional Algorithms classes using URI Online Judge, in proceedings of ICEE, pp. 110-113. 